\title{
Flow Characteristics of Drag-Reducing Surfactant Solutions
}

\author{
Takashi SAEKI* and Aya KAIDE \\ Graduate School of Science and Technology for Innovation, Yamaguchi University, 2-16-1 Tokiwadai, Ube, Yamaguchi \\ 755-8611, Japan
}

\begin{abstract}
The objective of this study is to clarify the flow characteristics of drag-reducing flow and to elucidate the mechanism underlying this phenomenon. The surfactant and counter ion we used were Lipothoquad $\mathrm{O} / 12$ and sodium salicylate, respectively. The drag reduction rate $(D R \%)$ was measured by using a recirculating system with a diameter of $25.6 \mathrm{~mm}$. We also measured the flow characteristics of the surfactant solutions with different concentrations and temperatures by using particle image velocimetry (PIV). From the experimental results, $D R \%$ at an average velocity of $2.0 \mathrm{~m} / \mathrm{s}$ increased from 0 to $68 \%$ as the temperature increased from 10 to $40{ }^{\circ} \mathrm{C}$ at a constant concentration of surfactant $(300 \mathrm{mg} / \mathrm{L})$. From the velocity contour plot obtained from PIV, we found that the thickness of the lower-velocity region of the drag-reducing flow near the pipe wall was thick at $20^{\circ} \mathrm{C}$, whereas vortex motions seemed controlled at $30^{\circ} \mathrm{C}$. On the other hand, the lower-velocity region thickened as the concentration of the surfactant increased at $25^{\circ} \mathrm{C}$. Even if the same level of drag-reducing effects occurred, the flow patterns were quite different depending on the concentration and temperature.
\end{abstract}

\section{Introduction}

It is well known that certain cationic surfactant solutions with counter ions significantly reduce drag in turbulent flow. Among them, since the cationic surfactant oleylbishydroxyethyl-methyl-ammonium chloride (brand name: Lipothoquad $\mathrm{O} / 12$ ) shows a sufficient and stable drag-reducing effect, it has been adopted at more than 200 sites in air-conditioning systems throughout Japan, including office buildings, hotels, hospitals, supermarkets, airport facilities, and industrial factories (Saeki and Tokuhara, 2014). In comparison with the coherent structure of normal turbulence, the vortex motion of drag-reducing flow can be restrained by the rheological properties of surfactant solutions, greatly changing flow patterns as a result. Many PIV (particle image velocimetry) studies using CTAC (cetyltrimethylammonium chloride) as a cationic surfactant have been reported (Gu et al., 2010, 2012; Kawaguchi et al., 2002; Li et al., 2008); however, there are insufficient data for Lipothoquad $\mathrm{O} / 12$. Also, the effects of the concentration of the surfactant and the solution temperature on the drag-reducing effect are not fully understood in connection with the coherent structure of the flow. In this study, PIVs of surfactant systems were measured with varying concentrations and solution temperatures. The objective of our study was to clarify the flow characteristics of drag-reducing flow and to elucidate the mechanism underlying this phenomenon.

\section{Experimental}

\subsection{Drag reduction experiment}

The cationic surfactant used was Lipothoquad O/12 (oleyl-bishydroxyethyl-methyl-ammonium chloride, $\left.\mathrm{C}_{18} \mathrm{H}_{35} \mathrm{~N}^{+}\left(\mathrm{CH}_{3}\right)\left(\mathrm{C}_{2} \mathrm{H}_{4} \mathrm{OH}\right)_{2} \mathrm{Cl}^{-}\right)$produced by Lion Specialty Chemicals, Japan. The counter ion selected was sodium salicylate $\left(\mathrm{HOC}_{6} \mathrm{H}_{4} \mathrm{COO}^{-} \mathrm{Na}^{+}\right.$, called $\mathrm{NaSal}$ ). The experimental apparatus used for evaluating drag reduction was a recirculation system as shown in Figure 1. The surfactant and counter ion were added to a tank with a weight ratio of 5:3, and solutions were prepared. The temperature of each solution was controlled by using a heater and cooling water system with a temperature control device (Model E5CS, Omron, Japan). The flow rate was controlled by an inverter system installed to a main pump to the desired flow rate between $0-81 \mathrm{~L} / \mathrm{min}$. The flow rate was measured by an electromagnetic flow meter (Model FD-83, Keyence, Japan). A pressure drop test section was a straight PVC pipe measuring $1600 \mathrm{~mm}$ long and $25.6 \mathrm{~mm}$ in inside diameter. Measurements were carried out by an electric differential manometer MT210, Yokogawa, Japan). The Reynolds number was calculated with the property of water. The friction factor, $f$, was calculated as follows:

$$
f=\frac{1}{4} \Delta P\left(\frac{D}{L}\right)\left(\frac{1}{\rho u_{\mathrm{av}}^{2} / 2}\right)
$$

where $\Delta P, D, L, \rho$, and $u_{\text {av }}$ were the pressure drop, pipe diameter, pipe length, density of water, and average velocity, respectively. $u_{\mathrm{av}}$ was calculated from measured flow rate dividing by the cross-sectional area of the

\footnotetext{
* Corresponding author: saeki@yamaguchi-u.ac.jp
} 
straight PVC pipe. For convenient comparison of the drag reduction results between water and the test solutions, the drag reduction rate, $D R \%$, was defined as

$$
D R \%=\frac{f_{w}-f_{s}}{f_{w}} \times 100
$$

where $f_{w}$ and $f_{s}$ were the friction factor of water and that of solution, respectively. $f_{w}$ was obtained by using the Blasius equation:

$$
f=0.0791 R e^{-0.25}
$$

From the definition, $D R \%$ can be zero for water flow, while the positive values of $D R \%$ indicate the reduction percent of surfactant solutions with respect to the water flow. Negative values of $D R \%$ occurred sometimes in the lower flow rate range, which caused a higher viscosity of a surfactant solution than that of water. Since the viscosities of surfactant solutions show complex functions of shear rate, it is commonly used the viscosity of water for the calculation of Reynolds number. This method clearly shows the deviation from water flow and thus directly the magnitude of drag reduction, which is useful for application (Ohlendorf et al., 1986).

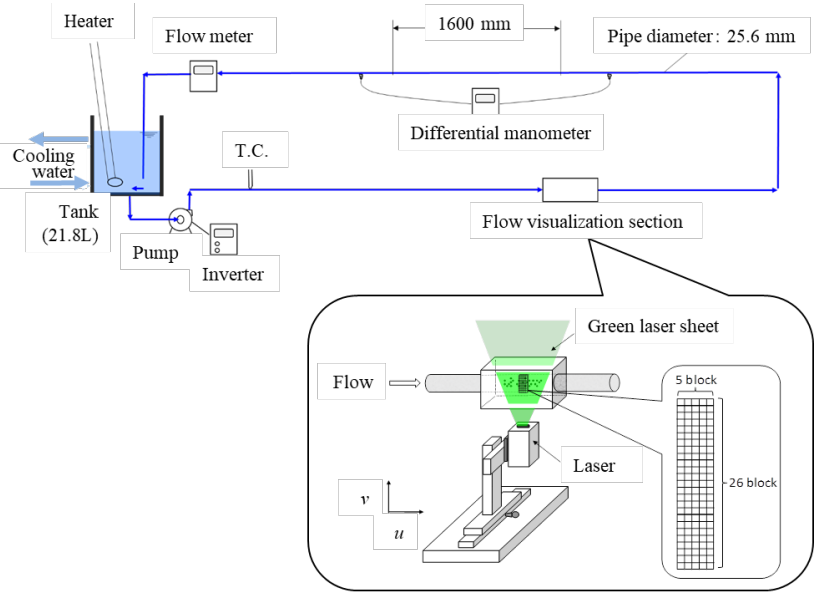

Figure 1. Experimental apparatus.

\subsection{PIV}

Polyvinyl chloride particles with an average diameter of $150 \mu \mathrm{m}$ were added as tracer particles to the test solutions. A flow visualization section at a point $3.0 \mathrm{~m}$ from the inlet of the straight test section consisted of an $80 \mathrm{~mm}$ transparent polyvinyl chloride pipe with a wall thickness of $0.2 \mathrm{~mm}$. The section was illuminated through a $2-\mathrm{mm}$ slit by using a green laser sheet and visualized by a high-speed video camera (VW-6000 motion analyzing microscope, Keyence, Japan) with a shutter speed of $2000 \mathrm{fps}$. Analyses of the obtained images over a region of $60 \times 30 \mathrm{~mm}$ were conducted by using PIV software (Flow Expert 32, ver.1.2.12, Katoh
Koken, Japan). The region was subdivided into $5 \times 26$ blocks. The axial and radial velocity data for each block were accumulated. The time-averaged axial velocity data were used to draw velocity profiles, while instantaneous velocity vectors for the region were displayed as contour charts.

\section{Results and Discussion}

\subsection{Drag reduction}

Figure 2 shows the friction factors and the Reynolds numbers for solutions with different concentrations at $25^{\circ} \mathrm{C}$. The drag reduction increased with the Reynolds number and reached a maximum point. When the Reynolds number was further increased, the drag reduction was lost rather abruptly, which may have been caused by the breakup of the surfactant micelleaggregate structures due to high shear. With an increasing surfactant concentration, the maximum drag reduction shifted to a higher Reynolds number.

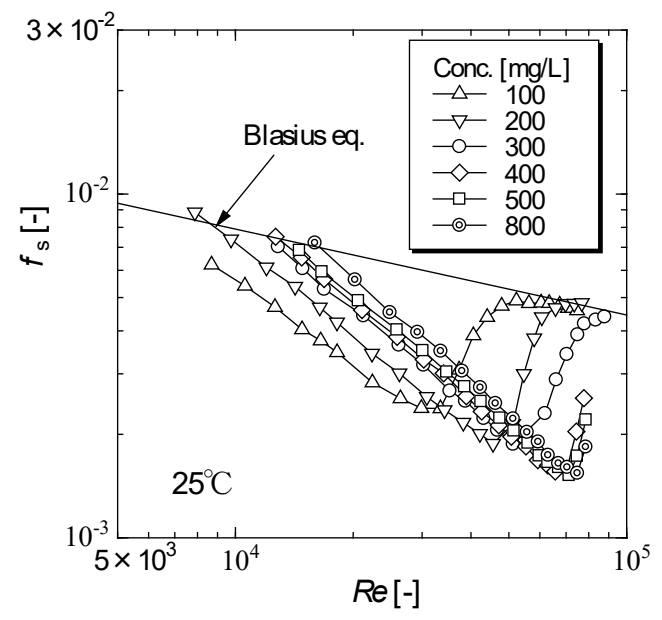

Figure 2. Drag reduction results for Lipothoquad $\mathrm{O} / 12$ and $\mathrm{NaSal}$ systems at different concentrations at $25^{\circ} \mathrm{C}$

Figure 3 shows $D R \%$ versus $u_{\mathrm{av}}$ for the same experiment. The maximum $D R \%$ for each solution increased with the increase of the concentration and reached an almost constant value of around $65 \%$ at concentrations of more than $400 \mathrm{mg} / \mathrm{L}$.

Figure 4 shows $D R \%$ versus $u_{\mathrm{av}}$ for $300 \mathrm{mg} / \mathrm{L}$ Lipothoquad $\mathrm{O} / 12$ solutions at different temperatures. The maximum $D R \%$ for each temperature was shifted to a higher average velocity with increasing temperature.

Whereas the average velocity of the water plumbing of an air conditioner is generally designed to be 1-1.5 $\mathrm{m} / \mathrm{s}$ (economical velocity), the velocity of a heat transfer pipe of a heat exchanger is more than $2 \mathrm{~m} / \mathrm{s}$ in order to enhance the heat transfer due to turbulence. Therefore, it is important to elucidate the drag-reducing flow in this border in consideration of the analogy between the momentum and heat transfer of the flow. From this point of view, $D R \%$ values at the average velocity of $2.0 \mathrm{~m} / \mathrm{s}$ obtained for the above-mentioned experiments are listed 
in Table 1 from the central focus at $300 \mathrm{mg} / \mathrm{L}$ and $25^{\circ} \mathrm{C}$. The increase in $D R \%$ with respect to the concentration may be related to the strength of the viscoelasticity of the solution, which may contribute to the relaxation of the turbulence. Solution temperatures also relate to $D R \%$; however, the strength of the viscoelasticity of a solution generally decreases with the increase in solution temperature. Such a contradiction may be explained by introducing the flow property of drag-reducing flow.
Table 1. $D R \%$ at the average velocity of $2.0 \mathrm{~m} / \mathrm{s}$ for each temperature and concentration.

\begin{tabular}{llrrrr}
\hline & \multicolumn{2}{c}{ Temp. } & & & \\
Conc. & 10 & 20 & 25 & 30 & $40{ }^{\circ} \mathrm{C}$ \\
\hline $100 \mathrm{mg} / \mathrm{L}$ & & & 6 & & \\
200 & & & 38 & & \\
300 & 0 & 43 & 53 & 66 & 68 \\
400 & & & 66 & & \\
500 & & & 64 & & \\
800 & & & 61 & & \\
\hline
\end{tabular}

\subsection{Flow property}

\subsubsection{Velocity distribution}

Figure 5 displays the velocity distribution of water measured at the average velocity of $2.0 \mathrm{~m} / \mathrm{s}$ by using the PIV apparatus, where $u, r$, and $R$ indicate the local averaged velocity, the distance from the pipe center, and the radius of the pipe, respectively. Our results fit well with the Power law.

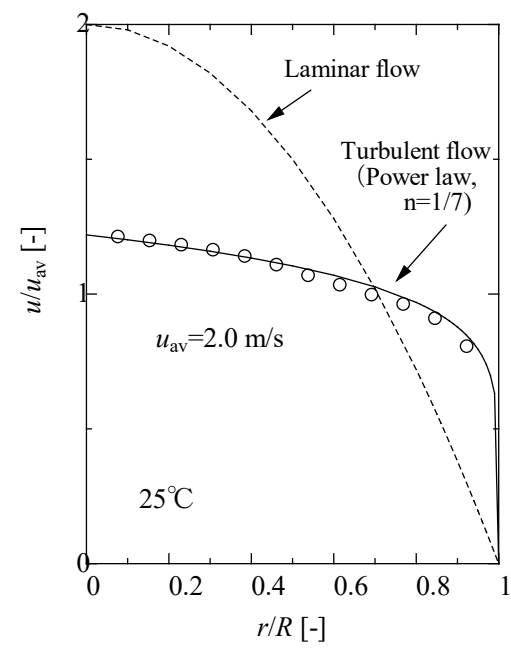

Figure 5. Velocity distribution of water measured in this study.

Figure 6 (a) shows the velocity distributions of the solutions at the average velocity of $2.0 \mathrm{~m} / \mathrm{s}$ with varying concentrations. Velocities near the center of the pipe decreased with the increase in the concentration up to $500 \mathrm{mg} / \mathrm{L}$. At the same time, velocities in the range of $r / R=0.6-0.7$ increased in order to store the flow rate. However, the trend of the distribution at $800 \mathrm{mg} / \mathrm{L}$ was different even though the solution showed similar levels of drag reduction. Figure 6 (b) displays velocity distributions with varying temperatures. Again, velocities near the center of the pipe decreased when drag was reduced; however, we could not discern a organized tendency. 


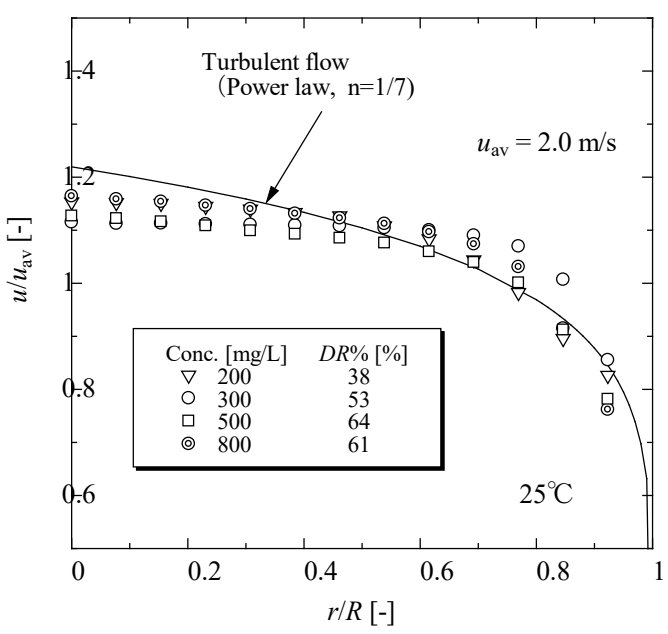

(a) Concentration dependence.

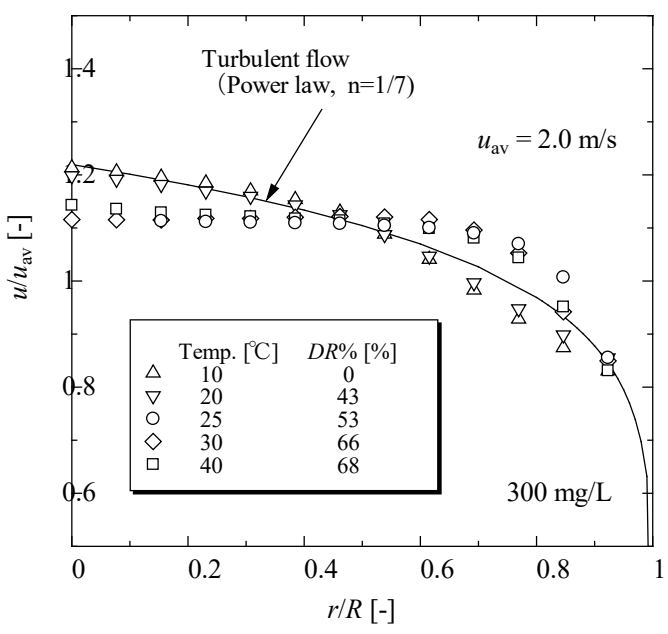

(b) Temperature dependence.

Figure 6. Velocity distribution of Lipothoquad O/12 and NaSal systems at an average velocity of $2.0 \mathrm{~m} / \mathrm{s}$

Figure 7 shows a contour chart obtained for water flow at an average velocity of $2.0 \mathrm{~m} / \mathrm{s}$. The turbulent core (red to green portions) and the boundary layer region (blue and sky-blue) were confirmed.

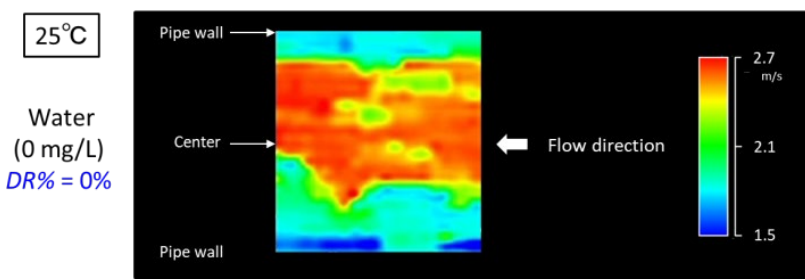

Figure 7. Contour chart of velocity vector obtained for water at an average velocity of $2.0 \mathrm{~m} / \mathrm{s}$
Figure 8 show contour charts of solutions at an average velocity of $2.0 \mathrm{~m} / \mathrm{s}$ with varying concentrations. The coherent structure for $100 \mathrm{mg} / \mathrm{L}$ was not similar to that of water even when $D R \%$ was $0 \%$. The lowervelocity region near the wall became thin and/or vague at $200 \mathrm{mg} / \mathrm{L}$, whereas the region almost disappeared at $300 \mathrm{mg} / \mathrm{L}$, where $D R \%$ was $53 \%$. On the other hand, although the higher (redder) velocity region expanded at $800 \mathrm{mg} / \mathrm{L}$, the lower-velocity regions can also be seen near the wall.
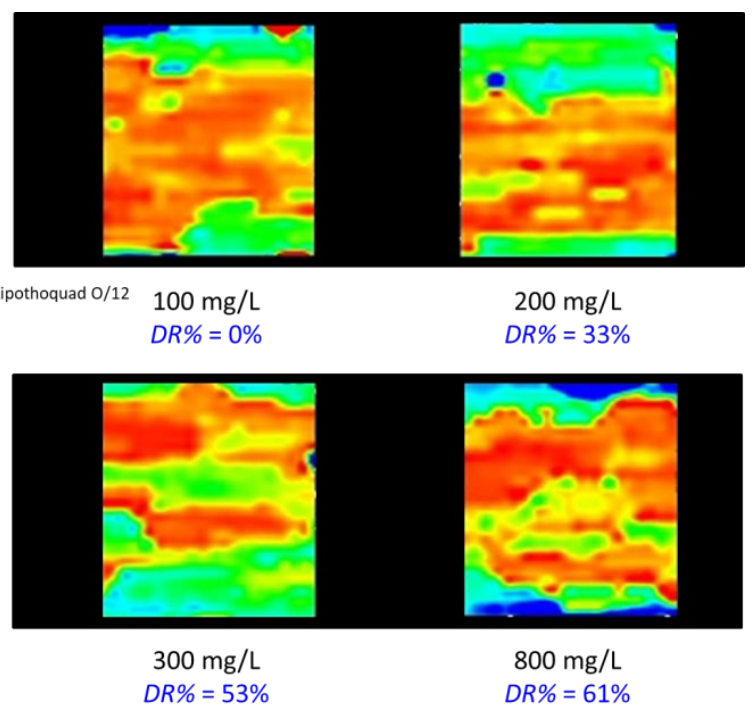

Figure 8. Contour charts of the velocity vector obtained for Lipothoquad $\mathrm{O} / 12$ and $\mathrm{NaSal}$ systems at different concentrations. The temperature and average velocity were $25^{\circ} \mathrm{C}$ and $2.0 \mathrm{~m} / \mathrm{s}$, respectively.

Figure 9 show contour charts of $300 \mathrm{mg} / \mathrm{L}$ solution with varying temperatures. Although the coherent structure for $10^{\circ} \mathrm{C}$ showed a thick boundary layer near the wall, the image of the turbulent core region was not similar to that of water even when $D R \%$ was $0 \%$. The lower-velocity regions became thin at $25^{\circ} \mathrm{C}$ and almost disappeared at $30^{\circ} \mathrm{C}$. We found that the coherent structure at $30^{\circ} \mathrm{C}$ was considerably different from that at $800 \mathrm{mg} / \mathrm{L}$ in Figure 8 even though both solutions showed remarkably similar levels of drag reduction.

The mechanism underlying drag reduction cannot be regarded simply as the reduction of friction between the fluid and the pipe wall. The reason why $D R \%$ can take significantly large values might be explained by performing some kind of control of turbulent eddies near the wall. In this study, we found at least two patterns of coherent structures for drag reducing flows:

1) The lower-velocity region near the wall became thin, vague, and/or disappeared compared with water flow.

2) The higher-velocity region of the core expanded and a low-speed domain presented near the wall.

Although both flow patterns significantly reduced drag, the heat transfer characteristics must be different due to 
the presence of a large vortex near the wall. It will be necessary to clarify the difference experimentally in a future study. We suggest that the drag reduction can be clarified by Lagrange-type analysis, not by analysis based on statistics such as velocity distribution obtained by averaging the local velocity.

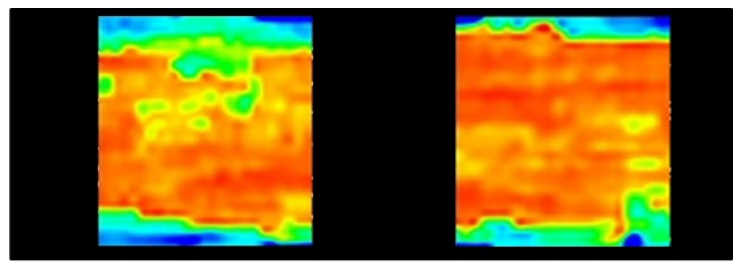

$10^{\circ} \mathrm{C}$

$D R \%=0 \%$

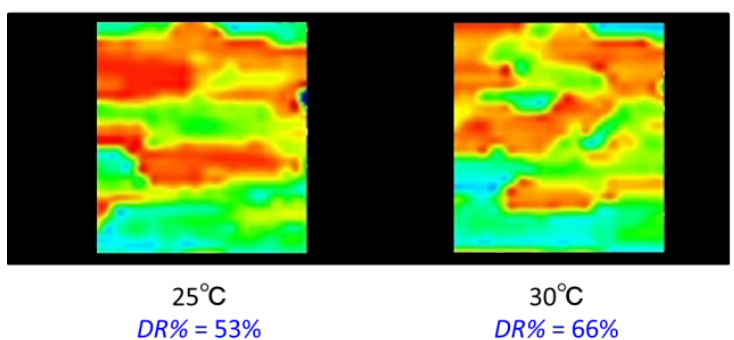

Figure 9. Contour charts of the velocity vector obtained for Lipothoquad $\mathrm{O} / 12$ and NaSal systems at different temperatures. The concentration and average velocity were $300 \mathrm{mg} / \mathrm{L}$ and 2.0 $\mathrm{m} / \mathrm{s}$, respectively.

\section{Conclusions}

Surfactant drag-reducing flow was examined by changing both the concentration of surfactant and the temperature of the solutions. The drag reduction rate $(D R \%)$ was measured by using a recirculating system, while the flow characteristics were measured by using particle image velocimetry. From the experimental results, we observed at least two patterns of coherent structures for drag-reducing flow. We pointed out that even when the same level of drag-reducing effects was taking place, the flow patterns differed significantly depending on the concentration and temperature of the solutions.

\section{References}

Gu, W.-G., Y. Kawaguchi, D. Wang, and A. Saito; "Experimental Study of Turbulence Transport in a Dilute Surfactant Solution Flow Investigated by PIV," J. Fluids Eng., 132, 051204 (2010)

Gu, W.-G. and D. Wang; "Turbulence Transport of surfactant Solution Flow during Drag Reduction Degeneration,", J. Hydrodynamics, Ser. B, 24, 479-487 (2012)

Kawaguchi, Y., T. Segawa, Z. Feng, and P. Li; "Experimental Study on Drag-Reducing Channel Flow with Surfactant Additives-Spatial Structure of Turbulence Investigated by PIV System," Int. J. Heat Fluid Flow, 23, 700-709 (2002)

Li, F.-C., Y. Kawaguchi, B. Yu, J. Wei, and K. Hishida; "Experimental Study of Drag-Reduction Mechanism for a Dilute Surfactant Solution Flow," Int. J. Heat Mass Transfer, 51, 835-843(2008)

Ohlendorf, D., W. Interthal, and H. Hoffmann; "Surfactant Systems for drag-Reduction: PhysicoChemical Properties and Rheological Behaviour," Rheologica Acta, 25, 486-486 (1986)

Saeki, T. and K. Tokuhara; "Development and Spread of Energy Saving Technology for Fluid Transportation by Using Drag-Reducing Effect," (in Japanese) Nihon Reoroji Gakkaishi, 42, 279-284 (2014) 